Revue d'histoire de l'Amérique française

ZRS REVUE D.HISTOIRE DE L'AMÉRIQUE FRANÇAISE

\title{
Le Québec et le monde communiste : Cowansville 1931
}

\section{Andrée Lévesque}

Volume 34, numéro 2, septembre 1980

URI : https://id.erudit.org/iderudit/303854ar

DOI : https://doi.org/10.7202/303854ar

Aller au sommaire du numéro

Éditeur(s)

Institut d'histoire de l'Amérique française

ISSN

0035-2357 (imprimé)

1492-1383 (numérique)

Découvrir la revue

Citer cet article

Lévesque, A. (1980). Le Québec et le monde communiste : Cowansville 1931.

Revue d'histoire de l'Amérique française, 34(2), 171-182.

https://doi.org/10.7202/303854ar d'utilisation que vous pouvez consulter en ligne.

https://apropos.erudit.org/fr/usagers/politique-dutilisation/ 


\title{
LE QUÉBEC ET LE MONDE COMMUNISTE: COWANSVILLE 1931
}

\author{
ANDRÉE LÉVESQUE \\ Département d'histoire \\ Université d'Ottawa
}

Au début de mars 1931, un "peddler» arrive à Montréal des Cantons de l'Est et entre en contact avec le directeur de la Ligue de la Défense ouvrière (LDO) pour l'informer d'une grève qui vient de se déclarer parmi les travailleurs de la soie à Cowansville. La grève a été déclenchée le 28 février quand la Compagnie a avisé les tisserands d'une réduction de salaire de $25 \%$. Quatre-vingt-dixneuf travailleurs - 76 hommes et 23 femmes - débrayent; les divisions de la teinture et de l'imprimerie, soit 148 employés, ne seront pas en grève mais leur travail, qui dépend de celui des tisserands, sera néanmoins affecté. Henry Segal, organisateur syndical et membre de la LDO, transmet la nouvelle au district communiste numéro deux ${ }^{1}$, et le lendemain matin un jeune communiste originaire d'Angleterre, Tom Miller, prend le train pour Cowansville. Le soir même, c'est-à-dire le trois mars, un membre de la Ligue des Jeunesses communistes, Fred Rose, va le rejoindre. Quelques jours plus tard on comptera cinq organisateurs communistes: Fred Rose, Tom Miller, Don Chalmers, Jeanne Harvey (Corbin) et Emery Simard, en contact avec les grévistes de la Bruck Silk Mill, le plus important employeur du village de 1800 habitants.

Qui sont ces délégués communistes qui se donnent pour mission de noyauter la grève? On comprend le choix de Fred Rose et de Don Chalmers, âgés de 27 et 29 ans; tous deux sont actifs dans la Ligue des Jeunesses communistes et ils s'intéressent particulièrement à l'industrie textile, dont la main-d'oeuvre est très jeune. La Ligue estime en effet que 70 à $80 \%$ des travailleurs du textile sont

1 Pour fins administratives, le Parti Communiste du Canada était divisé en neuf régions géographiques ou districts. Le Québec tout entier formait le district numéro deux, par contre, l'Ontario comprenait quatre districts. Voir Ivan Avakumovic, The Communist Party in Canada. A History (Toronto, McClelland and Stewart Ltd., 1975), 32.

RHAF, vol. 34, no 2, septembre 1980 
des «jeunes $»^{2}$. Rose, Chalmers et Miller attendent leur procès sous une accusation de sédition et d'incitation à l'émeute à la suite d'arrestations effectuées le 19 janvier 1931 lors d'une réunion du Conseil des Sans-Travail à Montréal ${ }^{3}$. La présence de Jeanne Harvey est tout aussi indiquée. Cette ex-institutrice, organisatrice infatigable et éditeur de L'Ouvrier canadien, est bon orateur et a l'avantage de pouvoir communiquer avec les grévistes dans leur langue. Simard, le cinquième envoyé, est un forgeron qui s'était porté candidat aux élections fédérales de 1930 dans Maisonneuve et avait recueilli 317 voix $^{4}$.

Les cinq compagnons vont rencontrer un conflit de travail assez typique pendant la grande crise économique. Les grèves dans l'industrie textile ne sont pas rares pendant les années trente, mais il est de prime abord étonnant qu'elles surviennent dans l'industrie de la soie naturelle. En raison de la hausse du tarif et de l'effondrement des prix de la soie brute, la valeur et le volume de la productioñ aùgmeñteñt à partir de $\mathbf{i} 930$. La réduction de salaire y est inférieure à celle que l'on connaît dans l'industrie manufacturière en général. Pourtant, malgré la situation privilégiée des usines de soie, les salaires y demeurent inférieurs à ceux de l'industrie du coton. Dans les établissements québécois qui, comme la Bruck Silk Mill, emploient de 100 à 200 ouvriers, ils sont encore plus bas ${ }^{5}$. À la Bruck les salaires varient de 13 cents l'heure pour les jeunes filles de moins de 18 ans, à 29 cents pour les hommes de plus de 21 ans ${ }^{6}$. Sans syndicat, sans fonds de grève, les ouvriers sont dans une situation très difficile. Certains d'entre eux, des jeunes qui viennent de la campagne environnante, peuvent éviter le pire en retournant vivre chez leurs parents. Les hommes mariés qui habitent Cowansville dépendent beaucoup du bon vouloir de la Compagnie.

Les causes de cette grève n'ont rien de bien spécial pour cette période. Mais une chose la distingue et lui confère un intérêt particulier: la présence d'organisateurs communistes. On ne saurait

2 APO, Att.-Gen. Files, boîte 11, env. 26, 11 C 2581.

3 Tous trois feront éventuellement un stage à la prison de Bordeaux pour sédition, après quoi Chalmers sera déporté en Écosse.

4 Le Devoir, 29 juillet 1930.

5 Procès-verbaux, Commission Royale d'Enquête sur les Écarts des Prix, no 35, mardi, 18 décembre 1934 (Ottawa, Imp. du Roi, 1934), 2721. - Rapport de la Commission Royale d'Enquête sur les Écarts des Prix (Ottawa: Imp. du Roi, 1935), 90 et 418.

6 Procès-verbaux, Commission Royale d'Enquête sur les Écarts des Prix, no 35, mardi, 18 décembre 1934 (Ottawa, Imp. du Roi, 1934), 2737. 
comprendre les activités communistes pendant l'ère staliniste sans se référer à la Troisième Internationale Communiste (IC, Komintern) dont la ligne d'action est dominée par les impératifs de la politique soviétique. Les Congrès de l'IC énoncent la ligne qui devra être suivie sur les différentes scènes internationales de Canton à Paris, en passant par les centres moins névralgiques comme Toronto.

En 1931, l'orientation idéologique et stratégique du Parti communiste du Canada (PCC) est déjà établie depuis le VIe Congrès de l'IC en 1928. Plus particulièrement, elle a été appliquée au Canada au VIe Congrès du PCC, en 1929. Le Komintern a pour priorité d'assurer le succès du socialisme en URSS d'abord, soit la réalisation du Plan quinquennal ainsi que la défense contre les attaques militaires, économiques et politiques des puissances capitalistes. Le programme de l'IC, enfin rédigé et approuvé par le VIe Congrès, établit les devoirs de ses membres envers l'URSS. Au-delà de leurs aspirations nationales, les prolétaires du monde entier doivent donc garantir l'établissement de la citadelle socialiste soviétique et se soumettre à la discipline internationale communiste ${ }^{7}$. Émus des conséquences de la rationalisation du travail pour les masses ouvrières, convaincus de l'imminence d'une guerre impérialiste qui portera le coup de grâce au capitalisme, sensibilisés au danger de la montée du fascisme, et surtout inspirés par les querelles intestines entre trotskyistes, boukharinistes et stalinistes, les délégués réunis à Moscou pendant l'été 1928 décident de donner un virage à gauche aux partis communistes: on refusera désormais toute collaboration avec les socio-démocrates, qui sont devenus des sociaux-fascistes, et le mot d'ordre «classe contre classe» exprime ce durcissement ${ }^{8}$. On poursuit la «bolchévisation» des partis communistes.

Les directives du Komintern sont transmises sur la scène canadienne lors du VIe Congrès du PCC en juin $1929^{9}$. Le mouvement ouvrier, l'implantation dans les usines, doivent former la base du Parti. Avant ce Congrès une politique d'infiltration dictait aux militants d'adhérer aux syndicats internationaux et nationaux en

7 Institute of Marxism-Leninism, CC of CPSU, Outline History of the Communist International (Moscou, Progress Publishers, 1971), 275-280.

8 Pour le Sixième Congrès, voir Annie Kriegel, «La Troisième Internationale», dans J. Droz, Histoire générale du Socialisme, III: 92-100.

9 Voir I. Avakumovic, chap. 3: 54-95. 
favorisant toutefois ceux du Congrès pan-canadien du Travail, et de les radicaliser.

La débâcle économique ne fit que confirmer les prédictions de certains leaders communistes et c'est trois semaines après le krash que l'on fonda la Ligue de l'Unité ouvrière (ou Ligue Unitaire ouvrière, LUO), section canadienne de l'Internationale ouvrière, ou Internationale Rouge, Profintern, qui aura pour tâche spécifique l'organisation ouvrière ${ }^{10}$. Tom Ewen (McEwen) en sera le secrétaire national à Toronto et chaque district aura sa section de la LUO. Dans son Manifeste de l'Exécutif National de novembre 1929 , sans toutefois abandonner totalement ses efforts d'infiltration des syndicats en place, la Ligue demande à ses membres d'accorder priorité à la formation d'unions industrielles dans les industries non-organisées et là où les syndicats sont les moins actifs $\mathrm{s}^{11}$.

$\mathrm{Au}$ Canada, comme aux États-Unis d'ailleurs ${ }^{12}$, le Parti recrute ses membres surtout parmi les minorités nationales: Finlandais, Allemands, Hongrois, Slâves s'y retroúveint ei il arrive aussi souvent que les réunions se passent en yiddish. En 1930, il compterait plus de 2900 membres au Canada; en 1931, un rapport du district no 2 établit le total des membres au Québec à 238, dont la moitié sont en règle, et dont une vingtaine seulement sont francophones $^{13}$. Le PCC se propose de diriger ses efforts vers le recrutement des Canadiens, anglophones et francophones. Cette décision est d'autant plus pertinente pour le Québec que, d'après la thèse selon laquelle le Canada est un pays impérialiste, s'y concentrent les masses les plus exploitées par l'impérialisme canadien ${ }^{14}$. Au double point de vue de leur importance numérique

10 «Profintern Resolution on the Canadian Situation and Tasks of the W.U.L. of Canada, 1930», APO, Att.-Gen. files, boîte 10, env. 19, 10 C 1909-1916; J. Gray, "The First W.U.L. Congress and its Tasks in Canada», Inprecor, no 32: 134-135.

11 APO, Att.-Gen. files, boîte 9, env. 8, 9 C 0832.

12 Pour la situation aux États-Unis, voir N. Glazer, The Social Basis of American Communism, chap. 4, «Jews and Middle Class Groups, and the Party» (N.Y.: Harcourt, Brace \& World Inc., 1961). J.S. Salsberg, militant communiste pendant cette période, commentera plus de trente ans plus tard: «Workers of Foreign birth, the Canadians of recent arrivals, they of course suffered the evils of unemployment and low wages perhaps more than the old established workers. It stands to reason that they should have been more militant and seek support for militant organizations. Also many brought with them a more mature understanding of socialism and perhaps of socialist membership in the countries for which they came.» Entrevue avec Paul Fox, 22 mai, 196[?] APC, Paul Fox Papers, MG 31, K3.

13 Une campagne de juin à août, 1931, portera le total à 200, dont 50 francophones. «Resolution of Organisation Conference District \#2, CPC», Att.-Gen. files, boîte 8, 8 C 0027-8.

14 «Lessons of the 6th Convention», The Worker, 14 décembre 1929. - The Worker, 22 novembre 1930. 
et de leur infériorité économique, les Québécois font l'objet de la plus grande sollicitude de la part du Parti: la LUO assume donc comme principal objectif l'organisation de «200000 ouvriers francophones» ${ }^{15}$.

En 1930, le Congrès du Profintern passe quelques résolutions sur la situation canadienne et sur la LUO au Canada. On accuse de «gauchisme» certains militants canadiens responsables de grèves perdues qui découragent les ouvriers, dit-on, et les laissent à la merci des «bureaucrates des unions réformistes et socialesfascistes $»^{16}$. On voit ici la position difficile des militants canadiens qui doivent danser sur une corde raide en évitant de tomber d'une part dans l'opportunisme qui dilue le message révolutionnaire dans cette période de "classe contre classe», et d'autre part dans l'extrémisme de gauche qui décourage les travailleurs. En tant que section du Profintern, la LUO réclame le renversement du capitalisme mais la persistance de la crise économique force la Ligue à accorder une importance croissante au maintien des salaires, à l'amélioration des conditions de travail et aux mesures de sécurité sociale. On réclame donc des réformes immédiates tout en essayant de ne pas perdre de vue la restructuration économique qu'on relègue à un futur moins immédiat.

Alors que les opérations nationales sont dirigées de Toronto, siège du Comité central du Parti et du secrétariat de la Ligue, les activités québécoises sont concentrées à Montréal et, au bureau de la rue Ste-Catherine, se réunissent les principaux groupes d'action communiste: agitation-propagande, LUO, la section féminine et la presse. C'est de là qu'on distribue l'organe du Parti, The Worker, publié à Toronto. Grâce aux efforts de Jeanne Harvey, à partir de mai 1930 s'y ajoutera la publication de l'Ouvrier canadien «pour l'établissement d'un gouvernement Ouvrier et Paysans (sic) au Canada» ${ }^{17}$. La LUO, qui relève de Tom Ewen de Toronto, a sa propre organisation à l'intérieur du Parti, spécifiquement pour appliquer la politique d'organisation industrielle: conscientiser et encadrer les travailleurs, francophones surtout, chez qui le taux de syndicalisation est relativement bas ${ }^{18}$. Elle a pour objectif de

15 APO, Att.-Gen. files, boîte 9, env. 8, 9 C 0835.

16 Ibid., boîte 10, env. 19, 10 C 1909-1916.

17 L'Ouvrier canadien, 15 mai 1930.

18 Au Québec, en 1931, on compte 72144 syndiqués sur 696339 employés à gages. Jacques Rouillard, Les Syndicats nationaux au Québec de 1900 à 1930 (Québec, Les Presses de l'Université Laval, 1979), 234. Annuaire Statistique Québec, 1935 (Québec, Imprimeur du Roi, 1935), 91. 
rejoindre les travailleurs à l'intérieur des usines et ateliers, mais vu les effectifs limités des communistes et le chômage qui sévit, cette tactique n'est pas applicable à une grande échelle. Un autre plan d'action consiste à noyauter une grève en cours, soit en se mêlant aux lignes de piquetage, soit en prêtant ses talents d'organisateurs. La grève à Cowansville illustre ce type d'action.

Rendus sur place, les organisateurs communistes tenteront d'agir à l'intérieur du comité de grève déjà mis sur pied avant leur arrivée, et d'orienter leurs demandes dans l'optique de la LUO. Lors de l'assemblée des grévistes, ceux-ci tiendront des élections pour élargir leur comité et les représentants communistes essaieront, mais sans succès, d'y inclure des représentantes des grévistes féminines. Rose se présentera à l'assemblée en tant que membre de la LUO et tentera d'exposer les différences entre cette centrale et le Congrès pan-canadien du Travail, l'American Federation of Labor et la Confédération des Travailleurs catholiques du Canada. Ses attaques contre la centrale catholique ne passeront pas inaperçues et lui vaudront des interpellations d'un groupe de grévistes. L'assemblée élabore ensuite cinq demandes: 1) aucune réduction de salaire; 2) aucune amende pour la soie endommagée; 3 ) aucune discrimination contre les grévistes les plus actifs; 4) le congédiement des briseurs de grève; 5) la reconnaissance du syndicat ${ }^{19}$.

Quand le surintendant de la Compagnie refuse de rencontrer un comité composé de 20 membres, on dépêche trois délégués et tous les grévistes se massent devant l'usine. L'entrevue se solde quand même par un échec et la Compagnie annonce qu'elle n'ouvrira pas ses portes la semaine suivante. Pendant la fin de semaine, de retour à Montréal, les communistes tiennent une réunion et décident de former un syndicat de l'Union industrielle des Travailleurs du textile et d'émettre des cartes de membre du Profitern.

La question des cartes utilisées pose un problème. On trouve la mention: «Section of the RILU», c'est-à-dire la Red International of Labour Unions, ou Profintern, suivie de:

The WUL (Workers Unity League, LUO) shall include all wage workers and their labor organizations in industry and agricul-

19 Fred Rose, «Report of the Cowansville Strike», 2, APO, Att.-Gen. files, boîte 2, env. 17, 2 A 0922-0924. 
ture, regardless of age, race, color or sex, who subscribe to its program of class struggle. ${ }^{20}$

Il est difficile de cacher son affiliation au mouvement communiste si l'on reconnaît être une section de l'Internationale Rouge. Réalisant l'effet d'une telle admission auprès de travailleurs déjà prémunis contre le danger communiste, Rose et Chalmers décident de biffer la mention du Profintern avant de distribuer les cartes $^{21}$. À Cowansville on émet plus de cent cartes aux grévistes et on organise la publication de feuillets à distribuer dans les petites villes de textile environnantes pour solliciter leur appui ${ }^{22}$.

Non moins important, on essaie de maintenir le moral des grévistes que la Compagnie essaie d'intimider. Il y a des rumeurs que la Compagnie peut rester fermée indéfiniment parce qu'elle a des stocks accumulés. Les chefs de grève voient imprimer au verso de leur chèque de paye:

"I, the undersigned... hereby acknowledge to have received this day from the Bruck Silk Mills, Limited the sum of $\$ \ldots$ in full payment and settlement of all claims for wages, salaries, bonuses or expenses due to me and in consideration hereof I do waive and renounce to any and all rights of action whatsoever which I may have against the said company. ${ }^{23}$

Le lundi 9 mars, ce n'est plus la grève mais le lockout. Au lendemain d'une forte tempête de neige, on monte une impressionnante ligne de piquetage, que traverseront seulement neuf briseurs de grève. À la réunion qui suit, cependant, on sent déjà vaciller certains grévistes qui, dit-on, auraient été visités par le curé. Ceux-ci suggèrent au comité de soumettre au patron leur acceptation d'une réduction de $121 / 2 \%$. Un discours de Rose va les en dissuader. Mais, le lendemain, le curé présente aux grévistes le résultat d'une entrevue avec leur employeur. Celui-ci maintient sa proposition d'une réduction de $25 \%$ pour le travail de jour et de $20 \%$ pour celui de nuit, sans prélèvement pour les dommages encourus, ni congédiement. Cette fois, malgré les efforts des membres de la

20 «Statement of the National Fraction of the WUL on the Cowansville (P.Q.) Strike Based on the Report of Comrade F. Rose», ler avril 1931, APO, Att.-Gen. files, boîte 10, env. 20, 10 C 2058 .

21 Rose, 2 A 0925.

22 Police Report, 23 mars 1931, no 547, APC, Min. du Travail, RG 27, vol. 347.

23 Fred Rose, 4, 2 A 0925. 
LUO, les grévistes acceptent les termes offerts par la Compagnie et ils reprendront leur travail le lundi suivant ${ }^{24}$.

Comment a-t-on appliqué les directives du VIe congrès à la Bruck Silk Mill? L'organisation, en passant par l'élection des comités de grève et l'élaboration de demandes, est conforme aux directives de l'Internationale ${ }^{25}$. Les attaques contre les syndicats internationaux et catholiques correspondent au front commun à la base. On s'en prend aux leaders et aux organisations ouvrières non-communistes tout en établissant des contacts avec les travailleurs ${ }^{26}$. Quant à la résistance des communistes au compromis qui aurait fait accepter des coupures de $12^{1} / 2 \%$, elle est tout à fait dans la ligne de l'IC. En effet, en 1930, le présidium reconnaissait dans les demandes «exagérées» un instrument de radicalisation: les travailleurs réalisant ainsi les limites du capitalisme ${ }^{27}$.

L'appartenance à une organisation révolutionnaire comme le Profintern pose toutefois des problèmes. Rose et Chalmers avaient décidé de biffer la mention de l'Internationale Rouge sur les cartes de membres pour ne pas rebuter les grévistes alors que pendant toute cette période, qui s'étend du VIe au VIIe congrès de l'IC en 1935, le PCC revendique la direction des grèves, l'organisation de chômeurs, et des manifestations publiques, sans chercher à minimiser son rôle ou à invoquer la spontanéité des participants. Le secrétaire de la LUO, Ewen, accusera Rose et Chalmers d'opportunisme pour vouloir dissimuler l'affiliation de la Ligue au Profintern. Sous-estimer le potentiel révolutionnaire des masses n'est-il pas une déviation de droite?

Dans une lettre à A. Rosenberg, organisateur de la LUO à Montréal, Ewen ne se fait pas d'illusions quant à la solution du conflit de Cowansville mais, comme le Parti n'a rien à perdre, il serait préférable, écrit-il, d'établir ses positions, de s'afficher en tant que communistes travaillant pour le bien-être des travailleurs. Il aurait donc fallu, selon Ewen, profiter de la grève pour conscientiser les travailleurs, leur montrer les avantages d'une organisation de grève révolutionnaire, et énoncer la politique du Parti et

24 Ibid.

25 «Extracts from the theses of the Xth ECCI Plenum on the Economic Struggle and the tasks of the communist parties, Sept. 1929», J. Degras, ed., The Communist International, 1919-1943, Documents (3 vols., Londres, Frank Cass \& Co., 1971), III: 58.

26 «Resolution of Enlarged ECCI Presidium on the Economic Crisis and Unemployment», Inprecor, 7 March, 1930, dans Degras, III: 108.

27 Meeting of Enlarged Presidium of ECCI», février 1930, Degras: 99. 
de la LUO. Les communistes auraient dû prendre la direction de la grève et ce, sans subterfuge, car il est impératif de bien établir la différence entre l'organisation communiste et celle des autres syndicats ouvriers ${ }^{28}$.

La discussion sur la tactique à adopter en de telles circonstances, s'afficher communiste et membre de l'Internationale Rouge, ou dissimuler son identité, se poursuit dans le Worker ${ }^{2}$ et aux réunions de la LUO à Toronto et à Montréal. Dans une lettre à Jeanne Harvey, Ewen est catégorique:

There are times in the Province of Quebec when for the sake of expediency, we will be compelled to hide the face of the Party to some extent, but we must never carry this into the Trade Union work and to score the R.I.L.U. off the cards or to hide the face of the revolutionary Trade Union movement, where the organized French workers are the victims of the most reactionary section of the Canadian Trade Union movement is absolutely impermissable... This is the rankest opportunism. ${ }^{30}$

La «fraction nationale» de la LUO présente aussi son rapport le premier avril et, en prenant en considération le rapport de Rose sur la grève, tempère quelque peu la critique de Ewen.

The extreme ideological backwardness of the French-Canadian workers and the pernicious influence of the R.C. church, which enters into every economic struggle on the side of the bosses and the capitalist state, while appearing to seek favours for the workers, renders it very necessary for our Party in the present stage of development in Quebec, to work carefully, even to the extent in certain instances and conditions, to conceal our identity from the main body of the workers for strategic and tactical reasons, but never losing sight of the necessity (sic) of building up around us in these struggles, a firm nucleus of the best elements that support our position. ${ }^{31}$

Ces deux prises de position diffèrent sensiblement. Si la «fraction nationale» appuie Rose et sa décision de cacher l'identité du Parti, le secrétariat national n'accepte aucun compromis dans l'organisation syndicale.

28 T. Ewen à A. Rosenberg, 10 mars 1931, APO, Att.-Gen. files, boîte 2, env. 17, 2A 0903; 9 mars 1931, 2 A 0908.

29 D. Chalmers, The Worker, 4 avril 1931.

30 T. Ewen à J. Corbin, n.d., boîte 2, env. 17, 2 A 0912.

31 «Statement of the National Fraction». 
Cette action microscopique illustre les difficultés auxquelles devaient faire face les militants de gauche pendant cette période de rigorisme idéologique. Les tensions qu'on décèle entre le bureau central à Toronto et le bureau de la Ligue à Montréal reflètent le conflit très sérieux qui déchire les PC américain et canadien au sujet de l' «American exceptionalism». Selon cette thèse, la situation nord-américaine se distingue de la situation prévalant en Europe et en Asie en ce que le capitalisme est si solidement ancré en Amérique du Nord que celle-ci se trouve à l'abri des crises économiques cycliques prévues par l'orthodoxie marxiste. À l'encontre du Komintern, les partisans de l' «American exceptionalism» prétendent que la capacité de rebondissement du capitalisme américain rend futiles les efforts révolutionnaires qui misent sur l'imminence d'une crise économique et une radicalisation des masses prolétaires. Cette déviation de droite est donc fustigée au VIe congrès de l'IC, ses protagonistes sont expulsés des PC américain et canadien, et la crise économique de 1929 tombe juste à point pour confïmer la décision du Komintern et sa vision apocalyptique du capitalisme $^{32}$.

Le PCC demeure extrêmement sensible au danger d' «exceptionalism» et l'attitude de Rose et Chalmers au Québec rappelle sans doute celle des dissidents américains et canadiens. Le souci de ne pas tomber dans cette erreur explique en partie l'intransigeance de Ewen envers le compromis au sujet des cartes de membres de l'Union industrielle.

L'acceptation de la discipline de fer est un élément essentiel de l'appartenance au Komintern. Selon le programme adopté par le VIe congrès, cette discipline implique «la subordination des intérêts partiels et locaux du mouvement aux intérêts généraux et permanents et la stricte application de toutes les décisions des organes dirigeants de l'Internationale communiste par tous les communistes ${ }^{33}$. En préservant les intérêts immédiats du Parti, en dissimulant son identité pour pouvoir s'attirer des membres, Rose et Chalmers ont transgressé cet article et méritent leur remontrance.

En période de dépression, avec une main-d'oeuvre disponible chez les fils de cultivateurs, la Compagnie peut se permettre de demeurer inflexible sans provoquer l'indignation de l'opinion

32 «Meeting of Enlarged Plenum of ECCI», 104.

33 Outline History of the Communist International, 279-280. 
publique. Malgré le nombre de travailleurs impliqués, la presse locale tend à minimiser l'ampleur de l'arrêt de travail. Le Sherbrooke Daily Record fait mention d'une petite grève contre des coupures de salaire mineures et rassure ses lecteurs, écrivant que si les grévistes ne retournent pas au travail ils seront facilement remplacés ${ }^{34}$. Il s'agit là d'un des rares échos de la grève. Les journaux de Montréal, de la région de Sherbrooke et de St-Hyacinthe, en font à peine mention, si ce n'est lors du retour au travail. $L a$ Patrie souligne quand même que les travailleurs ont perdu $\$ 18000$. de salaires en deux semaines ${ }^{35}$.

Il convient de s'attarder ici sur les diverses pressions exercées sur les travailleurs. Il ne faudrait pas considérer ceux-ci comme des pions passifs dans les jeux de la Compagnie, du clergé ou du Parti communiste. Les travailleurs ont pris l'initiative de priver la Compagnie de leur force de travail. Quelques tisserands, qui avaient déjà leur carte de membre de la United Textile Workers of America et qui avaient acquis une certaine expérience syndicale aux États-Unis, ont réagi sans tarder en élisant un premier comité de grève de sept membres et en élevant des lignes de piquetage aux portes de l'usine et à la gare, et cela avant l'arrivée des organisateurs de Montréal.

Quels que furent leurs contacts avec la LUO, ils n'en sont pas pour autant sympathiques au communisme. Dès l'arrivée de Miller et Rose, ils s'informent du syndicat que ceux-ci représentent. À plusieurs reprises, ils dénoncent ce qu'ils appellent «l'esclavage des travailleurs en URSS» et accueillent avec suspicion les dénonciations de l'AFL et des syndicats catholiques. Dans une industrie employant une assez forte proportion de travailleuses, celles-ci jouent un rôle actif lors du piquetage. Elles essaient d'empêcher les briseurs de grèves et les seules arrestations seront celles de deux femmes qui en seraient venues aux coups avec les scabs ${ }^{36}$.

L'action du curé de la paroisse semble avoir été décisive. Le rapport de la police soumis au ministère du Travail observe de façon laconique: "The priest got the strikers to return to work». ${ }^{37}$ Le sermon du ler mars s'élève carrément contre la grève; le 8 mars, le curé demande aux grévistes de ne pas faire de trouble et de

\footnotetext{
34 Min. du Travail, RG 27, vol. 347.

35 La Patrie, 16 mars 1931 .

36 La Tribune de Sherbrooke, 4 mars 1931.

37 Police Report, 23 mars 1931, no 457, RG 27, vol. 347.
} 
laisser les briseurs de grèves aller à leur travail, "comme c'est leur droit $»^{38}$. Intermédiaire entre l'employeur et ses employés, le prêtre convaincra le comité de grève d'accepter les réductions de salaires.

Les jeunes membres de la LUO ont tout de même offert à un groupe de travailleurs québécois, dont plusieurs étaient en transition entre l'agriculture et l'industrie et partageaient leur temps entre la ferme et l'usine, leurs talents d'organisateurs, leur expérience en négociations patronales-syndicales, leur encouragement et leur support moral. Ils ont cependant laissé très peu de traces de leur passage: pas d'organisation, pas de leader pour prendre la relève, et surtout pas de gains matériels pour les travailleurs. Fidèles à l'orientation de l'heure, les militants auront, dans des circonstances fort peu propices, essayé de rejoindre les travailleurs québécois et de les conscientiser. Et si leurs efforts pour poser les bases d'une organisation se sont soldés par un échec, le Parti n'y a pas entièrement perdu puisqu'il aura fait son autocritique et tiré les leçons qui s'imposaient. Le débat provoqué par leuir interiventitioñ mêt ein relief le dilemme suscité par l'application de la doctrine communiste, qui est internationale, dans un contexte particulier fort éloigné des centres de décision. 